\title{
236 In a child with a tracheostomy
}

A Endoscopy prior to decannulation is useful rather than essential in long standing cases.

B There may be a predisposition to recurrent chest infections.

C A suprastomal granulation may result in decannulation difficulties.

D Surgical decannulation may result in surgical emphysema.

E The mortality of the procedure is about $1 \%$.

\section{Congenital abnormalities of the tracheobronchial tree}

A Account for less than $10 \%$ of cases of congenital stridor.

$B$ Include bulging of the posterior tracheal wall.

C Producing tracheomalacia are most commonly due to internal compression.

D Resulting in posterior tracheal compression occurs with an aberrant left subclavian artery.

E Vascular compression of the trachea with severe symptoms is best managed by tracheostomy.

\section{The following are indications necessitating tracheostomy}

A A child with acute laryngotracheobronchitis.

B Patients in whom endotracheal intubation is continued for more than about 3 weeks.

C A single failed attempt at endotracheal intubation in a child with acute supraglottitis.

D Total maxillectomy.

E Laryngeal trauma with evidence of frothy blood. 